\title{
The Sound of Communication in Underwater Acoustic Sensor Networks ${ }^{\star}$ (Position Paper)
}

\author{
Michel Barbeau ${ }^{1}$, Joaquin Garcia-Alfaro ${ }^{2}$, Evangelos Kranakis ${ }^{1}$, and Steven \\ Porretta $^{1}$ \\ 1 School of Computer Science, Carleton University, Ottawa, ON, Canada, K1S 5B6. \\ 2 Telecom SudParis, CNRS Samovar, UMR 5157, Evry, France.
}

\begin{abstract}
The interdisciplinary understanding required for the design and analysis of underwater acoustic sensor networks presents formidable constraints and challenges for computer scientists and engineers. However, underwater environments have never been much of a constraint to the rich animal life they support at all depths of our seas and oceans. Indeed, it is known that nature has taken advantage of this environment to develop a rich variety of efficient communication strategies throughout the long history of evolutionary change and adaptation. The wealth of knowledge to be discovered will continue to dazzle and fascinate the world. For the communications in underwater sensor networks, acoustic signaling is the preferred choice for designers because sound propagation is the most efficient when compared to other forms, like thermal, light, and electromagnetic. It is within this acoustic environment that researchers have to innovate and develop new ideas and methodologies so as to advance the state-of-the-art. In this position paper, several fundamental issues and connections are discussed that arise in the study of underwater wireless sensor networks. Throughout the nature of the underwater environment is emphasized how one can take advantage of it. A variety of ideas and solutions that could be of value for further research is proposed. Moreover, fundamental issues in topology control, directional underwater transducers, and monitoring and surveillance are discussed.
\end{abstract}

Key words: Underwater acoustic communications, directional hydrophone and vibrator, monitoring and surveillance, neighbour discovery, underwater acoustic sensor network, sub-surface communications.

\section{Introduction}

Sound is very important for communication in the animal world. It helps animals to become aware of events that occur all around, regardless of where attention is focused. With respect to their land counterparts, sea mammals are even more dependent on sound for communication and sensing because of the

\footnotetext{
* The research of Michel Barbeau and Evangelos Kranakis is supported in part by
} Natural Sciences and Engineering Research Council (NSERC) Discovery grants. 
special circumstances involved in the nature of signals underwater affecting the propagation of light, smell, and other senses. One must take into account that light propagation suffers from scattering due to reflection and refraction. Smell is affected by molecular diffusion due to temperature, viscosity of the fluid, and size of the particles. As a consequence, sight and smell could be ineffective and rather much less suited for communication in the seas when compared to sound. Sound has another advantage because water molecules lose less energy as they vibrate.

This paper explores how sound can be used to effectively communicate and build underwater networks. In Section 2, we introduce the nature of sound in the underwater environment. We explore the transmission of messages using sound in Section 3. A model for directional underwater communications is presented in Section 4. We conclude in Section 5.

\section{Nature of Communication Underwater}

You can calculate the speed $v(\mathrm{~m} / \mathrm{s})$ of sound by taking the square root of the ratio of the pressure $p(\mathrm{~Pa})$ of the medium, inside which it travels, divided by the density $\rho\left(\mathrm{kg} / \mathrm{m}^{3}\right)$ of this medium, namely

$$
v=\sqrt{\frac{p}{\rho}} \mathrm{m} / \mathrm{s} .
$$

In the air, it is approximately only $343 \mathrm{~m} / \mathrm{s}$ (at sea level). However, despite the fact that the propagation of sound underwater is affected by temperature, salinity, hydrostatic pressure and other factors, its speed in the ocean varies from $1,450 \mathrm{~m} / \mathrm{s}$ to $1,540 \mathrm{~m} / \mathrm{s}$. It is more than four times higher than its speed in the air. Also note how pressure affects the speed of sound. Approximately 1,6 $\mathrm{m} / \mathrm{s}$ per 100 meters downwards is added to the velocity due to the increase in hydrostatic pressure.

Mammalian evolution has created numerous adaptations so as to exploit the propagation of sound underwater. Acoustic communication in the seas is entirely different from the more familiar terrestrial. Moreover, in marine life, mechanisms used to produce sound vary widely even from one family of sea animals (such as whales, dolphins, and porpoises) to another. This is documented extensively in the scientific marine biology literature. For example, it is well known that the humpback whales are producing regular and predictable sounds known as songs to communicate male fitness to females. The clicking sequences of dolphins and sperm whales are thought to be individualized rhythmic sequences communicating the identity of a single mammal to others in its group. They allow groups to coordinate foraging activities. Furthermore, communication can reach large distances with sperm whales being the undisputed vocal champions that can give a powerfully deafening directional sonar of $240 \mathrm{~dB}$.

One cannot but marvel at the astonishing variety of sound based communication mechanisms that have evolved throughout sea life to communicate, attract mates, defend territory, sense surroundings and find food [4, 5, 8, 13]. See Ref. [24] 
where you can play recordings of all kinds of underwater animals, from whales and shrimps to oysters. Although whales can communicate long distance with their powerful sounds, at the opposite scale S. Patek [26,31] reports that the spiny lobster emits Near Field Communication (NFC) signals (that propagate no more than a meter) every time it throws off its exoskeleton. The very unique sound it generates (by using its body as a violin) protects the naked lobster against its enemies while at the same time the short distance of propagation prevents it from advertising its presence further away!

Underwater communication is not limited to sound. There are numerous surprising facts everywhere in the sea world. Electrical eels may have very poor eyesight, but they can stun their prey with their powerful electrical pulses like taser guns [8]. Turtles seem to inherit instructions for navigating through the earth's weak magnetic fields, travel long distances and return to their place of origin essentially performing a form of compass routing through the isoclinics and isodynamics of the earth's magnetic maps [22]. Also take note that magnetic navigation has the advantage that it works even in deep oceans where light is impossible to penetrate. In Australia, the archerfish (Toxotes chatareus) is an expert physicist in that it calculates the precise angle for shooting from inside the water to the air despite refraction to kill its prey [8]. Certain families of squid not only have directional antennae communication, but can also communicate with each other using their own morse code(see video in [21]). With their wonderful multicolor displays and convulsions, octopuses can match the color of their bodies instantaneously to their environment [10], presumably to transmit messages.

\section{Transmission of Sound}

What technical issues do we encounter in transmitting messages underwater? How can we take them into account and at the same time improve our communication capabilities? In this section, we discuss how sonar measurements are made underwater as well as the impact of waveguides (communication tunnels) for connectivity.

\subsection{Sonar Measurements}

Sonar (also called echolocation) refers to the principle of detecting and localizing objects by sound. When referring to animals, it is also called biological sonar or biosonar. Unlike the discovery of sonar in bats, which happened as early as 1773 (due to the Italian scientist Lazzaro Spallanzani), the discovery of sonar in dolphins did not occur until 1947 (due to Arthur McBride, the first curator of Marine Studios, in Florida). SONAR is an acronym for SOund Navigation And Ranging [3]. It is a technique that uses underwater sound propagation to navigate, communicate with or detect objects (such as submarines and mines) on or under the surface of the water by projecting sound and detecting the echoes from the objects. 
The key to measuring the intensity and pressure of acoustic waves is based on using the concept of decibel (dB). Since in underwater acoustics, the primary interest is often in ratios rather than in absolute quantities this gives a convenient way for expressing changes (usually large) in pressure. Given two powers $P_{1}$ and $P_{2}$ (Watts), with power ratio $P_{1} / P_{2}$, we use the decibel expression as given by the formula

$$
10 \log _{10}\left(\frac{P_{1}}{P_{2}}\right) \mathrm{dB}
$$

When an acoustic wave propagates in a medium, acoustic energy is being transmitted. The amount of energy per second crossing a unit area is called the intensity of the wave. The unit of intensity in underwater acoustics is defined as the intensity of a plane wave having a pressure $p$ of one micropascal $(\mu \mathrm{Pa})$. The relationship between acoustic pressure $p$ and intensity $I$ (Watts $/ \mathrm{m}^{2}$ ) is given by the formula

$$
I=\frac{p^{2}}{\rho v} \mathrm{Watts} / \mathrm{m}^{2}
$$

where $\rho\left(\mathrm{kg} / \mathrm{m}^{3}\right)$ is the density of water and $v(\mathrm{~m} / \mathrm{s})$ is the speed of sound.

The intensity ratio $I_{1} / I_{2}$ is defined in decibels similarly to the power ratio, i.e., the intensity ratio in $\mathrm{dB}$ is equal to

$$
10 \log _{10}\left(\frac{I_{1}}{I_{2}}\right) \mathrm{dB}
$$

The basic measurement in acoustics is based on pressure and not on intensity. Most hydrophones used in underwater measurements are sensitive to pressure, particle velocity, or pressure gradient. It follows from Equations (2) and (3) that the pressure ratio in decibels is expressed as $20 \log _{10}\left(P_{1} / P_{2}\right)$.

\subsection{Impact of Temperature and Pressure on Sound}

Underwater, propagation of sound is three dimensional. It propagates in all directions from its source. During transmission sound dissipates. Further, understanding its behaviour is complicated by such features as suspended particles, air bubbles, plankton, and even the swim-bladders of swimming fish.

The speed of sound in the ocean varies, see Equation (1). It depends on temperature, salinity, pressure and other factors. Note that the pressure $p(z)$ $(\mu \mathrm{Pa})$ is monotone increasing as a function of the depth $z(\mathrm{~m})$. Also temperature affects the speed $v(z)$ of sound, as a function of the depth $z$. The interplay of these two factors affect $v(z)$ that has a representation resembling the plot depicted in the left part of Figure 1. Closer to the surface, the speed $v(z)$ of sound is more affected by temperature. It decreases as we move deeper. As we move even deeper, the impact of pressure overtakes temperature. The speed of sound increases. Eventually, temperature and pressure balance out at a certain depth $z_{\min }$. The resulting speed of sound $v\left(z_{\min }\right)$ at $z_{\min }$ is the minimum possible. This depth $z_{\min }$ also depends on the oceanic temperature. It can be up to 2 kilometers in warmer waters while it is closer to the surface in the Arctic. 

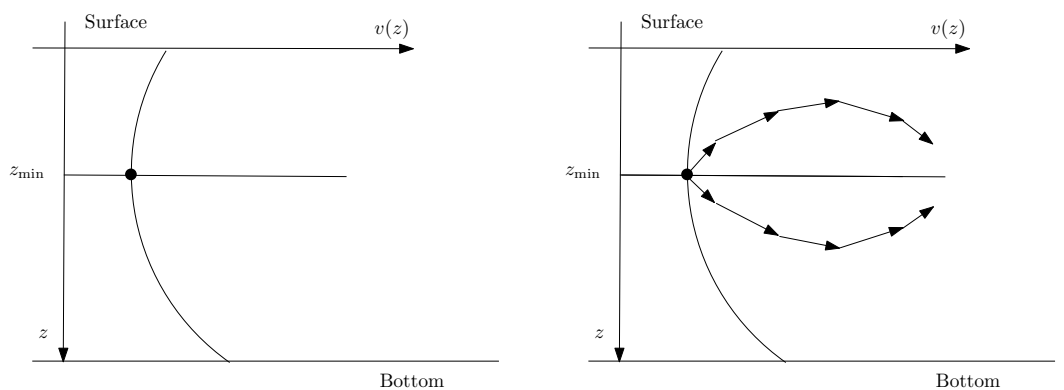

Fig. 1. Left Part: Diagram of the speed of sound $v(z)$ as a function of the depth $z$. Right Part: Due to refraction, a sound waveguide is formed whose walls delimit the propagation of sound when the emitting source is placed at depth $z_{\min }$.

\subsection{Impact of Refraction and Waveguides}

Sound propagating in the ocean can sometimes be detected thousands of kms away from the source. Does the ocean contain a channel (or acoustic waveguide) through which sound can propagate with little attenuation? Indeed, it is not difficult to speculate that a natural channel is formed between the surface of water and bottom of the ocean. But what mechanism do sound waves obey in such long-distance propagation? The basic principle is that transmission of sound along a waveguide is based on the reflection of waves along its boundaries which prevents scattering. It would seem as if sound propagates along a narrow tube reflecting along its boundaries. But how are such gigantic waveguides formed underwater and where are its boundaries?

It turns out that refraction plays a crucial role in the formation of waveguides. Assume a sound source placed at depth $z_{\min }$, see right part of Figure 1. Consider the sound beams emanating from it. Because of refraction, the propagation of the sound depends on the angle of the beam with respect to the horizontal. A beam propagating along a horizontal line is straight. A beam leaving $z_{\min }$ at an angle bends. However, since the speed of sound increases both up and down from the point $z_{\min }$, sound beams bend towards the horizontal. As a consequence this gives rise to a waveguide whose walls are formed by the layers of water at the depths where the sound beams reflect. For additional details see [2] (Chapter 3: The Oceanic Phone Booth) as well as S. Porretta's recent thesis [27].

To understand better the formation of waveguides, let $v(0)$ and $v(f)$ be the speeds of sound in the surface and bottom of the sea, respectively. It turns out that two types of waveguides may be formed depending on the relative sizes of the speeds $v(0)$ and $v(f)$.

Case $v(f)>v(0)$ : This usually occurs in deep water. On the one hand, when the water surface is calm the sound is reflected from the surface but is refracted at sea bed. In fact one can use Snell's law to determine what portion of the sound beam is captured by the channel [2]. On the other hand, when the water surface is rough the sound scatters from it. The rays leaving the surface at large 
angles reach the bottom and are absorbed there. However, because of refraction the channel captures those rays that do not reach the rough surface [2].

Case $v(f)<v(0)$ : This usually occurs in shallow water. In this case the sound refracted at the bottom does not reach the surface [2].

Schmidt and Schneider $[14,29,30]$ documented the existence of a waveguide in the Beaufort Sea, called the Beaufort Lens. Due to a flow of warm water entering through the Bering Strait, from the Pacific Ocean. A sound speed minimum is created at low depth, around $80 \mathrm{~m}$. Sound energy is trapped in the resulting waveguide. Long range (100 kilometers) communication, without ice interaction, is possible using the waveguide.

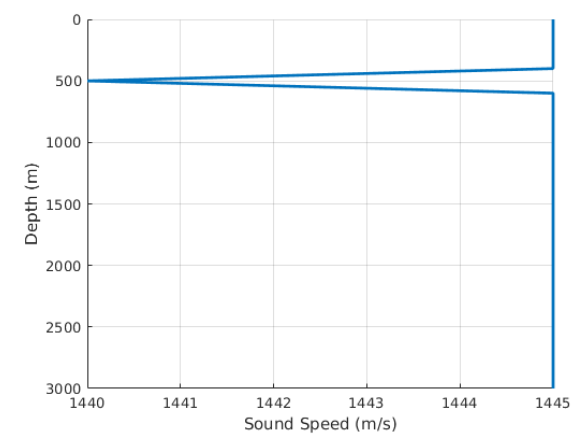

Fig. 2. Sound speed profile with local minimum.

The principle can be studied through simulation. Figure 2 shows a Sound Speed Profile (SSP), artificially created to better illustrate the idea. It plots the speed of sound ( $x$-axis) as a function of depth ( $y$-axis). There is a sound speed minimum at 500 meter deep. This minimum creates a waveguide in which the acoustic energy propagates without interaction with sea surface or sea bed. Figure 3 shows the result of a BELLHOP [28] simulation of the waveguide at the acoustic signal frequency 50 Hertz. The source-receiver separation distance is from zero to 100 kilometers. The $x$-axis represents range $(\mathrm{m})$. The $y$-axis represents depth. A signal source is placed on the left, at range zero and depth $500 \mathrm{~m}$. On a dB scale, color-coding represents signal attenuation as a function of location. In underwater, attenuation is proportional to frequency. Hence, attenuation is weaker on the left, staring at $70 \mathrm{~dB}$ on short range. The simulations demonstrate that signals propagating through a waveguide theoretically persist across long distances.

In conclusion, waveguides are an important component of establishing underwater communication. This, of course, should not come as a surprise given that also a similar phenomenon occurs in long-distance broadcasting from radio 


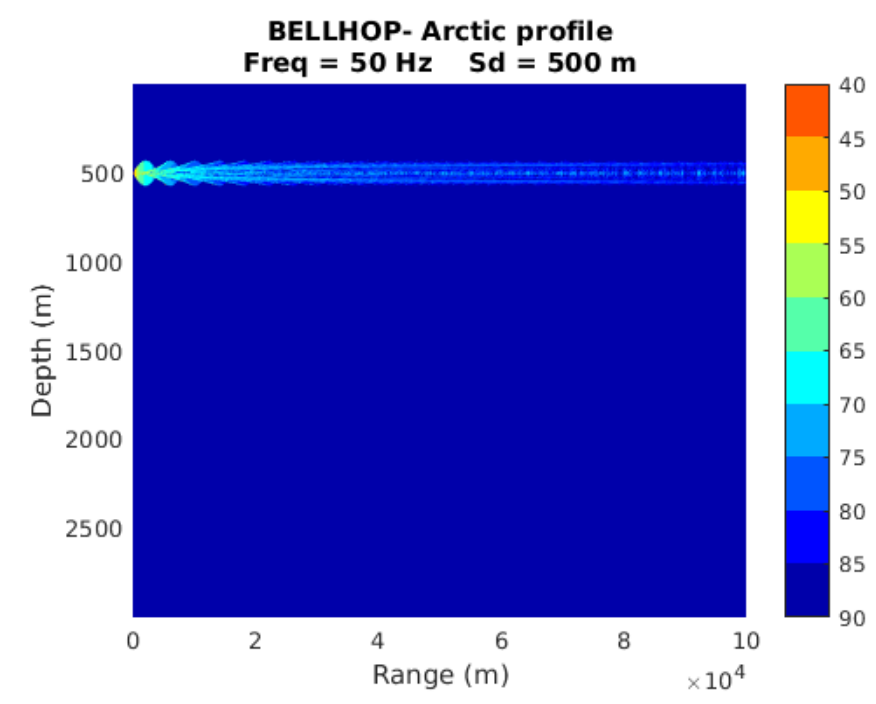

Fig. 3. Transmission loss (dB) versus distance and depth, at 50 Hertz and from zero to 100 kilometers.

stations. In fact, this is made possible only because of the propagation of radio waves through the atmosphere along giant waveguides.

\section{Directional Underwater Transducers}

Directional antennae are widely used in wireless communication. They are versatile. They improve overall energy consumption [18]. This is rather easy to motivate. The transmission cost is proportional to the area covered by the antenna. Thus, the energy cost of an omnidirectional antenna with range $r(\mathrm{~m})$ is proportional to the area of a disk of radius $r$, that is, $\pi r^{2} \mathrm{~m}^{2}$. By comparison the signal from a directional antenna of beam-width $\phi$ radians reaches much further, with the same energy consumption, namely it has range $r \sqrt{2 \pi / \phi} \mathrm{m}$ that, depending on the beam-width $\phi$, can be significantly larger than $r$. They have numerous applications. They may enhance network capacity $[12,33]$, reach further than omnidirectional antennae for detection and surveillance purposes, improve topology control and stability [11], and offer the potential for mitigating various security threats [15], just to mention a few applications.

A significant amount of research has been dedicated to the $2 \mathrm{D}$ model of directional antennae. In this model, the antennae are located in a planar terrain. To establish a network, antennae need to communicate with each other. To this end, two basic antennae communication models are employed. Consider two directional antennae: a sender and a receiver. In the symmetric model, communication is possible if the sender and receiver are within the range (determined by 
respective lobes) of each other, see $[1,23]$. In the asymmetric model, the sender can transmit directly a message to the receiver (provided the receiver is within the range of the sender) but the receiver may not be able to send directly a message to the sender, see $[6,9,17,20]$. In a way, the asymmetric model is less rigid than the symmetric one, but the receiver must seek a (alternate) path in the network if it also wants to talk to the sender.

\subsection{D Underwater Transducer Model}

Underwater communications differ from classical wireless. Rather than electromagnetic waves, mechanical acoustic waves are used. The transducers, converting electrical energy to mechanical energy and vice versa, are vibrators and hydrophones. Transmission is done with mechanical vibrators. Reception is done with mechanical hydrophones. Hereafter, we discuss a 3D underwater transducer model. We identify vibrators and hydrophones. We model a three dimensional
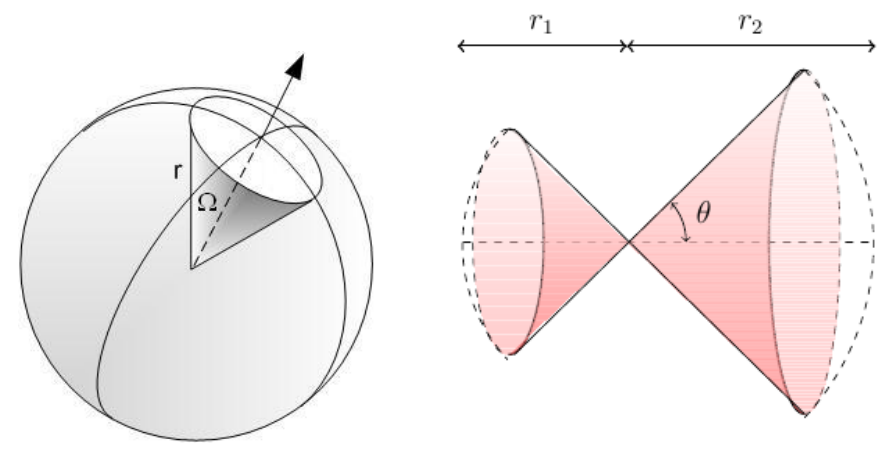

Fig. 4. Spherical underwater transducer radiation patterns. Solid (represented by $\Omega$ ) and apex (represented by $2 \theta$ ) angles in the $3 \mathrm{D}$ directional antenna model. The antenna in the left picture has a single lobe while in the right picture it has a double lobe.

directional underwater transducer as a spherical sector of solid angle $\Omega$ (Figure 4, left) and apex $2 \theta$ (Figure 4, right). The solid angle $\Omega$ of a solid spherical sector is defined as the ratio of the area of the spherical surface and square of the radius of the sphere of which it forms part. The apex angle of a spherical sector with solid angle $\Omega$ is defined as the maximum planar angle between any two generatrices of the spherical sector. It is usually represented by $2 \theta$, see [16] for additional details. The apex $2 \theta$ and solid angle $\Omega$ are related by the following important identity due to Archimedes.

$$
\Omega=2 \pi(1-\cos \theta)
$$


To see its validity note that, for a sphere of radius $r$, the area of the spherical cap satisfies

$$
\Omega=\int_{0}^{2 \pi} \int_{0}^{\theta} r^{2} \sin \theta^{\prime} d \theta^{\prime} d \phi=2 \pi r^{2} \int_{0}^{\theta} \sin \theta^{\prime} d \theta^{\prime}=2 \pi r^{2}(1-\cos \theta)
$$

Archimedes' Formula (4) gives a method to compute a 3D angle with the help of a $2 \mathrm{D}$ angle, see [32].

\subsection{Communication Model}

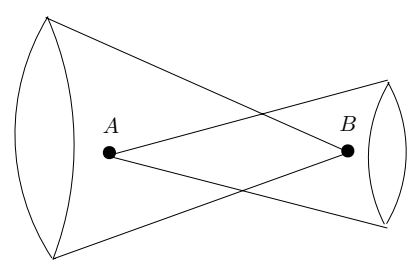

Fig. 5. Symmetric communication with two directional underwater transducers centered at points $A$ and $B$.

How can we model communication using directional underwater transducers? We distinguish two types of connectivity: symmetric and asymmetric. In symmetric communication, two underwater transducers communicate if they are within range of each other, see Figure 5. This also means they can send messages directly to each other. Contrast this with asymmetric communication (Figure 6)

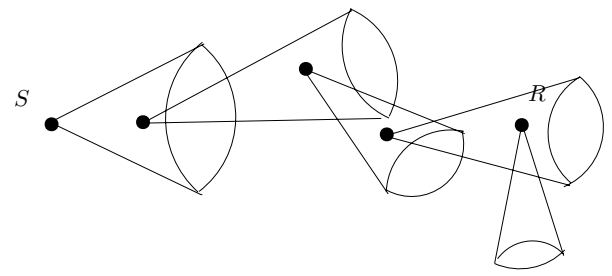

Fig. 6. Aymmetric communication with directional underwater transducers: A directed communication path from the vibrator $S$ to the hydrophone $R$.

in which a vibrator $S$ can talk to a hydrophone $R$ only if there exists a sequence of (vibrator, hydrophone) pairs $S \rightarrow S^{\prime}$ such that

$$
S:=S_{1} \rightarrow S_{2}, S_{2} \rightarrow S_{3}, \ldots, S_{k-1} \rightarrow S_{k}:=R
$$


and moreover so that each hydrophone in this sequence is within the range of a vibrator. Thus, to establish bidirectional communication between $S, R$ in the asymmetric communication model not only a path must be found between source $S$ and destination $R$; in addition, a path must be found in the reverse direction from destination $R$ to source $S$. Despite this difficulty it is still possible to provide algorithms that can establish bidirectional communication $[6,9,17$, 20] with constant stretch factor.

\subsection{Neighbour Discovery}

How does a underwater node discover its neighbour(s)? The neighbour discovery process usually entails the exchange and subsequent confirmation of identities between two adjacent nodes so that they can identify each other. To achieve this, omnidirectional underwater transducers must be, at a minimum, within range of each other (so that they can receive each other's messages). Thus, neighbour discovery for directional underwater transducers in the symmetric model requires not only that they must be facing each other but also be within range of each other. However, in the asymmetric model a communication path must be found between a sender and a receiver. Regardless of the communication model being used, the underwater nodes must execute an algorithm to discover their neighbour(s). To simplify matters, let us look first at 2D. Consider two directional

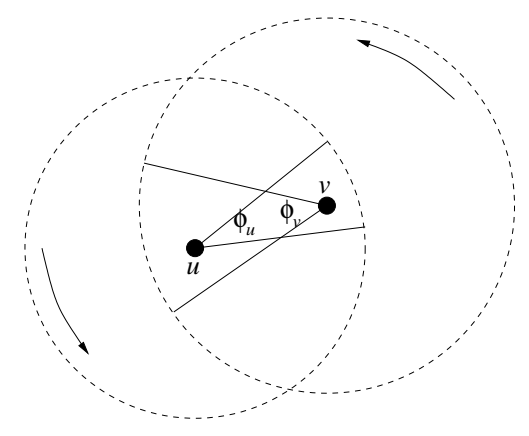

Fig. 7. Neighbour discovery for two underwater nodes that are within range of each other.

nodes $u$ and $v$ with beam-width $\phi_{u}$ and $\phi_{v}$, respectively. To communicate their underwater transducer must be facing each other, see Figure 7 . For each node $u$, let $d_{u}$ be an integer delay parameter and $k_{u}$ be defined so that $\phi_{u}=\frac{2 \pi}{k_{u}}$ and consider Algorithm 1. 


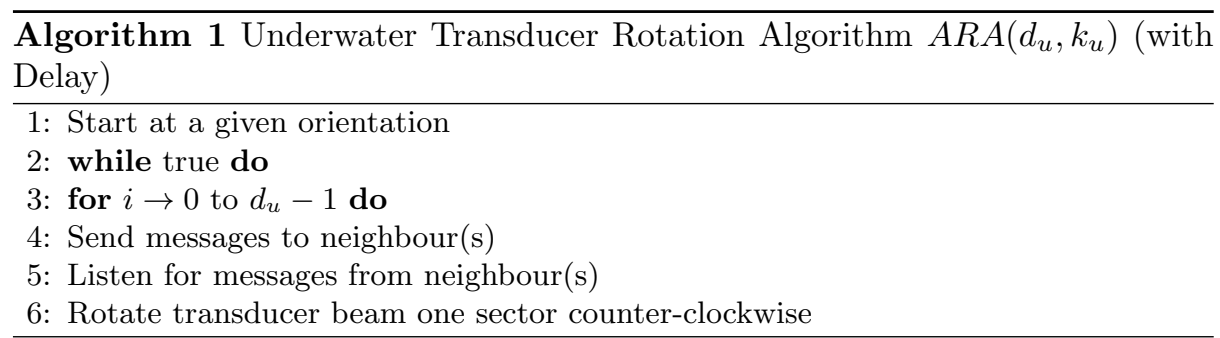

It can be shown (see [7] for details) that for a set $S$ of synchronous nodes by appropriately choosing (either deterministically or at random) the delay $d_{u}$, for $u \in S$, Algorithm 1 ensures that every pair of underwater transducers within range will discover each other.
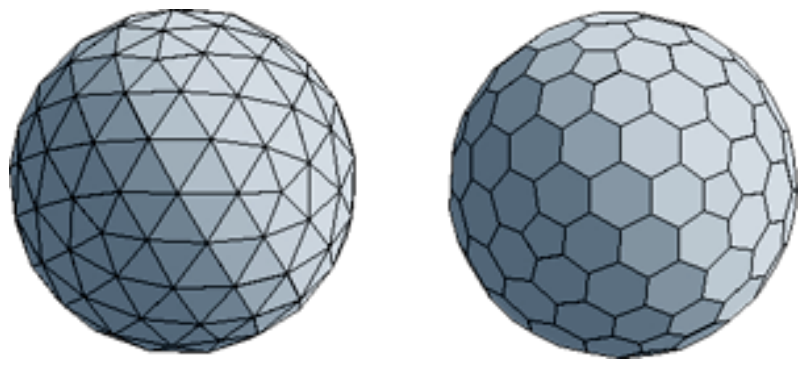

Fig. 8. Triangular and Hexagonal space partitions.

An approach to solving the neighbour discovery problem for underwater nodes is to adapt the previous approach, except that underwater transducer rotations must be done in 3D. The key idea is to use a partition of 3D space in a geodesic grid (see Figure 8) centered at each underwater node. Moreover, like in Algorithm 1, underwater transducers would somehow have to rotate over a well-defined domain specified by a geodesic grid (see Figure 8). The rotation mechanism (speed and direction of rotation) may depend on some knowledge of the environment and on the depth (see also [19] for a related study).

\subsection{Monitoring and Surveillance}

A potential application would be in establishing a wireless underwater networked system of communicating nodes to form an effective monitoring and surveillance network. Figure 9 depicts a linear array of underwater hydrophones and a passing autonomous vehicle. Each hydrophone has the ability not only to detect a passing autonomous vehicle, but also to discover its neighbours and transmit messages to them (other nodes within its range). Further, and unlike the scheme proposed 


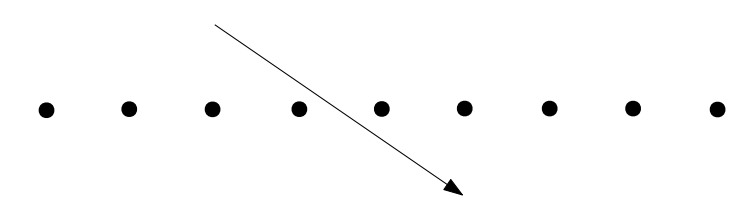

Fig. 9. An array of hydrophones and a passing underwater autonomous vehicle.

by [25] which is static and not immune to transducer failures, the resulting array is dynamic and fault tolerant thus also adapting to a changing communication environment.

\section{Conclusion}

In this paper, we discussed the rich communication capabilities of underworld life that have evolved over millions of years and the inspiration they may provide for new and more effective underwater network designs. We also considered some of the main impediments to establishing underwater communication networks and how they can they be mitigated. Research in UWANs (Under Water Acoustic Networks) requires a multidisciplinary approach involving scientists and engineers of widely varying academic backgrounds, experience and expertise.

Several questions and possibilities were discussed in this paper. We looked at some characteristics of underwater communication and how they can be used so as to develop methodologies for better and more reliable connectivity. Further, we discussed the possibility of designing a wireless networked system based on underwater hydrophones to support monitoring and surveillance services. The ultimate goal would be to aid the design of surveillance underwater wireless acoustic networks in (harsh) underwater environments.

\section{References}

1. R. Aschner, M. J. Katz, and G. Morgenstern. Symmetric connectivity with directional antennas. Computational Geometry, 46(9):1017-1026, 2013.

2. L. Aslamazov and A. Varlamov. The wonders of physics. World Scientific Publishing Co Inc, 2012.

3. Whitlow W. L. Au. The sonar of dolphins. Springer Science \& Business Media, 2012.

4. J. Balcombe. What a fish knows: The inner lives of our underwater cousins. Macmillan, 2016.

5. J. Tyler Bonner. Why size matters: from bacteria to blue whales. Princeton University Press, 2011.

6. I. Caragiannis, C. Kaklamanis, E. Kranakis, D. Krizanc, and A. Wiese. Communication in wireless networks with directional antennas. In SPAA 2008: Proceedings of the 20th Annual ACM Symposium on Parallelism in Algorithms and Architectures, Munich, Germany, June 14-16, 2008, pages 344-351, 2008. 
7. J. Du, E. Kranakis, O. Morales Ponce, and S. Rajsbaum. Neighbor discovery in a sensor network with directional antennae. In International Symposium on Algorithms and Experiments for Sensor Systems, Wireless Networks and Distributed Robotics, pages 57-71. Springer, 2011.

8. M. Durrani and L. Kalaugher. Furry Logic: The Physics of Animal Life. Bloomsbury Sigma, 2017.

9. M. Eftekhari Hesari, E. Kranakis, F. MacQuarrie, O. Morales Ponce, and L. Narayanan. Strong connectivity of sensor networks with double antennae. Theor. Comput. Sci., 610:192-203, 2016.

10. P. Godfrey-Smith. Other Minds: The Octopus, The Sea, and the Deep Origins of Consciousness. Farrar, Straus and Giroux, 2017.

11. H. Gupta, U. Kumar, and S.R. Das. A topology control approach to using directional antennas in wireless mesh networks. IEEE International Conference on Communications, 9(06):4083-4088, 2006.

12. P. Gupta and P. R. Kumar. The capacity of wireless networks. Information Theory, IEEE Transactions on, 46(2):388-404, 2000.

13. P. Hoare. The Whales In Search of the Giants of the Sea. Harper Collins, 2010.

14. T. Howe. A modal analysis of acoustic propagation in the changing arctic environment. Master's thesis, Massachusetts Institute of Technology. Department of Mechanical Engineering, 2016.

15. L. Hu and D. Evans. Using directional antennas to prevent wormhole attacks. In Network and Distributed System Security Symposium (NDSS), 2004.

16. E. Kranakis, D. Krizanc, A. Modi, and O. Morales Ponce. Connectivity trade-offs in $3 \mathrm{~d}$ wireless sensor networks using directional antennae. In 25th IEEE International Symposium on Parallel and Distributed Processing, IPDPS 2011, Anchorage, Alaska, USA, 16-20 May, 2011 - Conference Proceedings, pages 345-351, 2011.

17. E. Kranakis, D. Krizanc, and O. Morales Ponce. Maintaining connectivity in sensor networks using directional antennae. In Sotiris E. Nikoletseas and José D. P. Rolim, editors, Theoretical Aspects of Distributed Computing in Sensor Networks, pages 59-84. Springer (Monographs in Theoretical Computer Science.), 2011.

18. E. Kranakis, D. Krizanc, and E. Williams. Directional versus Omnidirectional Antennas for Energy Consumption and k-Connectivity of Networks of Sensors. In proceedings of OPODIS 2004, LNCS, 3544:357-368, 2005.

19. E. Kranakis, F. MacQuarrie, I. K. T. Maffra, and O. Morales Ponce. Strong connectivity of wireless sensor networks with double directional antennae in 3d. In International Conference on Ad-Hoc Networks and Wireless, pages 257-268. Springer, 2013.

20. E. Kranakis, F. MacQuarrie, and O. Morales Ponce. Connectivity and stretch factor trade-offs in wireless sensor networks with directional antennae. Theor. Comput. Sci., 590:55-72, 2015.

21. J. J. Lee. Jumbo squid speak by 'flashing' each other". National Geographic, January 21, 2015, http://news.nationalgeographic.com/news/2015/01/150121humboldt-jumbo-squid-crittercam-animal-ocean-science/. Accessed 2017-07-03.

22. K. J. Lohmann, S. D. Cain, S. A. Dodge, and C. M. F. Lohmann. Regional magnetic fields as navigational markers for sea turtles. Science, 294(5541):364-366, 2001.

23. R. Montemanni and L. M. Gambardella. Minimum power symmetric connectivity problem in wireless networks: a new approach. In Mobile and Wireless Communication Networks, pages 497-508. Springer, 2005.

24. University of Rhode Island Graduate School of Oceanography. Discovery of sound in the sea. http://www.dosits.org/. Accessed 2017-06-03. 
25. R. Otnes, J. E. Voldhaug, and S. Haavik. On communication requirements in underwater surveillance networks. In OCEANS 2008-MTS/IEEE Kobe TechnoOcean, pages 1-7. IEEE, 2008.

26. S. N. Patek. First-person: The benefits of "strange" science. Duke Magazine: http://dukemagazine.duke.edu/, March 14, 2016. Accessed 2017-07-03.

27. S. Porretta. Environmental communication optimization in underwater acoustic sensor networks, Masters Thesis in Computer Science, Carleton University, Spring 2017.

28. M. B. Porter. The BELLHOP manual and users guide: Preliminary draft, 2011.

29. A. Poulsen and H. Schmidt. Proceedings of the 22nd international congress on acoustics (ICA. In Acoustic noise properties in the rapidly changing Arctic Ocean, pages 1-4, Aug 2016.

30. H. Schmidt and T. Schneider. Acoustic communication and navigation in the new arctic; a model case for environmental adaptation. In 2016 IEEE Third Underwater Communications and Networking Conference (UComms), pages 1-4, Aug 2016.

31. E. R. Staaterman, T. Claverie, and S. N. Patek. Disentangling defense: the function of spiny lobster sounds. Behaviour, 147(2):235-258, 2010.

32. G. Van Brummelen. Heavenly mathematics: The forgotten art of spherical trigonometry. Princeton University Press, 2013.

33. S. Yi, Y. Pei, S. Kalyanaraman, and B. Azimi-Sadjadi. How is the capacity of ad hoc networks improved with directional antennas? Wireless Networks, 13(5):635$648,2007$. 